\title{
Ventajas del alfa ordinal respecto al alfa de Cronbach ilustradas con la encuesta AUDIT-OMS
}

\author{
Sergio Contreras Espinoza ${ }^{1}$ y Francisco Novoa-Muñoz ${ }^{1}$
}

Forma de citar Contreras Espinoza S, Novoa-Muñoz F. Ventajas del alfa ordinal respecto al alfa de Cronbach ilustradas con la encuesta AUDIT-OMS. Rev Panam Salud Publica. 2018;42:e65. https://doi.org/10.26633/ RPSP.2018.65

RESUMEN Objetivos. Exponer las ventajas de la utilización del alfa ordinal para situaciones en que no se cumplan los supuestos del alfa de Cronbach y mostrar la utilidad del alfa ordinal con la versión chilena del AUDIT, así como proporcionar los comandos en el lenguaje de programación $R$ para realizar los cálculos respectivos.

Métodos. Se compararon el alfa de Cronbach y el alfa ordinal en el caso del cuestionario AUDIT aplicado a 419 estudiantes de una universidad chilena.

Resultados. En todos los dominios, el alfa ordinal resultó ser mayor que el alfa de Cronbach, lo que es congruente con lo encontrado en la bibliografía. Al considerar el alfa ordinal se acepta la confiabilidad de cada uno de los dominios y de la encuesta completa, lo que no ocurre completamente al trabajar con el alfa de Cronbach.

Conclusiones. Para tomar decisiones sobre la confiabilidad de una dimensión se debe considerar un índice que cumpla los supuestos necesarios para su utilización, ya que no hacerlo puede desembocar en conclusiones erróneas y afectar de manera notable el desarrollo de una investigación. Los valores del alfa de Cronbach subestiman la confiabilidad de un instrumento al trabajar con escalas ordinales.

Palabras clave Consumo de bebidas alcohólicas; estudios de validación; confiabilidad; Chile.

Tradicionalmente, la validez se ha definido como el grado en que una prueba mide lo que está diseñada para medir (1). El alfa de Cronbach aparece con frecuencia en la bibliografía como una forma sencilla y confiable para realizar la validación de constructo de una escala y como una medida que cuantifica la correlación existente entre los ítems que la componen (2). Es el coeficiente más

\footnotetext{
Departamento de Estadística, Universidad del Bío Bío, Concepción, Región del Bío-Bío, Chile. La correspondencia se debe dirigir a Sergio Contreras. Correo electrónico: scontre@ubiobio.cl
}

ampliamente usado para estimar la confiabilidad en investigaciones aplicadas (3), pero, a pesar de su uso generalizado, su aplicación podría no ser correcta cuando la naturaleza de la escala de respuesta es ordinal, pues uno de los supuestos del coeficiente de Cronbach es la naturaleza continua de las variables (4).

Además, el uso del alfa de Cronbach o de cualquier otro coeficiente de confiabilidad, bajo circunstancias que violan sus supuestos o prerrequisitos, podría desembocar en su subestimación (5). De hecho, de forma más radical, el alfa de
Cronbach tiene una utilidad bastante limitada y se recomienda abandonarla (6).

Cuando no se cumplen los supuestos para la utilización del alfa de Cronbach al estimar la confiabilidad, una alternativa válida es el alfa ordinal, que no presenta las dificultades que tiene el alfa de Cronbach. A su vez, se afirma que se ha demostrado que el alfa de Cronbach subestima la magnitud de la confiabilidad cuando se tienen ítems con menos de cinco alternativas y se propone utilizar el alfa ordinal para escalas de respuesta binaria y ordinal (7). Además, se ha señalado que el alfa ordinal es conceptualmente

Este es un artículo de acceso abierto distribuido bajo los términos de la licencia Creative Commons Attribution-NonCommercial-NoDerivs 3.0 IGO, que permite su uso, distribución y reproducción en cualquier medio, siempre que el trabajo original se cite de la manera adecuada. No se permiten modificaciones a los artículos ni su uso comercial. Al reproducir un artículo no debe haber ningún indicio de que la OPS o el artículo avalan a una organización o un producto específico. El uso del logo de la OPS no está permitido. Esta leyenda debe conservarse, junto con la URL original del artículo. 
equivalente al alfa de Cronbach (5). La diferencia crítica entre estos dos coeficientes es que el alfa ordinal se basa en la matriz de correlación policórica, en lugar de la matriz de covarianza (correlación) de Pearson, y de esta forma es más adecuado para estimar alfa con mediciones con datos ordinales. También se destaca que el coeficiente de correlación de Pearson subestima gravemente la verdadera relación entre dos variables continuas cuando estas variables presentan asimetría. Otra propiedad importante del alfa ordinal es que es un estimador insesgado de la confiabilidad teórica para datos ordinales.

Varios estudios han sugerido la utilización del alfa ordinal, por lo que no es nuevo en la bibliografía, aunque su utilización es menos frecuente $(4,5,7)$. Una razón que podría explicar el poco uso del alfa ordinal es la dificultad para calcularla. Por este motivo, en el presente artículo se entregan los comandos en R (8), para realizar su cálculo de manera rápida y sencilla, aunque también pueden realizarse con Excel.

El objetivo del presente estudio es exponer las ventajas de la utilización del alfa ordinal para situaciones en que no se cumplen los supuestos del alfa de Cronbach y mostrar la utilidad de este coeficiente en el caso de la versión chilena del Alcohol Use Disorders Identification (AUDIT), aplicado a una muestra de estudiantes de una universidad chilena. En la referencia 9 se pueden ver los detalles de la versión AUDIT-Chile (9).

\section{MATERIALES Y MÉTODOS}

Existen varias investigaciones sobre el consumo de alcohol en estudiantes universitarios (10-17). En el caso de Chile, se estudiaron la validez y la confiabilidad de la versión de la Organización Mundial de la Salud (OMS) de AUDIT, usando el coeficiente alfa de Cronbach (que supone la continuidad de las variables) para conocer la confiabilidad del instrumento (cuyas respuestas son ordinales) (17). El instrumento AUDIT es una encuesta con escala de respuesta ordinal con algunos ítems con menos de cinco alternativas, por lo que es un ejemplo apropiado para mostrar las diferencias entre el alfa de Cronbach y el alfa ordinal.

En la presente investigación sólo se observaron los efectos de ciertas variables sobre el resultado del cuestionario
AUDIT. Por lo tanto, se trató de un estudio observacional descriptivo (18). El Cuestionario AUDIT es una prueba diseñada por la OMS en 1989 para detectar el consumo excesivo de alcohol y como un apoyo a los clínicos en la evaluación breve (19). El AUDIT consta de diez ítems. En él la escala de respuesta de las ocho primeras preguntas es 0,1 , 2, 3 y 4, siendo 0,2 y 4 la escala de respuesta de las últimas dos preguntas. Este instrumento contiene tres dimensiones, dominios o constructos para medir: el consumo de riesgo, los síntomas de dependencia y el consumo perjudicial de alcohol, que se definen a continuación.

El consumo de riesgo es un patrón de consumo de alcohol que aumenta el riesgo de consecuencias adversas para el bebedor o para los demás. Considera los ítems 1, 2 y 3 . La dependencia es un conjunto de fenómenos conductuales, cognitivos y psicológicos que pueden aparecer después del consumo repetido de alcohol. Considera los ítems 4, 5 y 6 . Y el consumo perjudicial se refiere a aquel que tiene consecuencias para la salud física y mental. Considera los ítems 6, 7, 9 y 10. En el cuadro 1 se muestran los dominios e ítems que componen cada constructo del cuestionario AUDIT.

El cuestionario AUDIT funciona a través de puntuaciones, donde el mínimo es 0 y el máximo es 40 puntos, que enmarcan a los individuos en distintas categorías. Se ha señalado que "A partir de la experiencia adquirida en la utilización del AUDIT en este y otros estudios de investigación, se ha sugerido que se debe dar la siguiente interpretación a las puntuaciones del AUDIT: puntuaciones entre 8 y 15 son las más apropiadas para un simple consejo enfocado en la reducción del consumo de riesgo, puntuaciones entre 16 y 19 sugieren terapia breve y un abordaje continuado, y

\section{CUADRO 1. Dominios e ítems del cuestionario AUDIT}

\begin{tabular}{lcl}
\hline Dominios & Pregunta & \multicolumn{1}{c}{ Contenido del ítem } \\
\hline Consumo de riesgo & 1 & Frecuencia de consumo \\
Síntomas de dependencia & 2 & Cantidad típica \\
& 3 & Frecuencia del consumo elevado \\
Consumo perjudicial & 4 & Pérdida del control sobre el consumo \\
& 5 & Aumento de la relevancia del consumo \\
& 6 & Consumo matutino \\
& 7 & Sentimiento de culpa tras el consumo \\
& 8 & Lagunas de memoria \\
\hline
\end{tabular}

Fuente: Referencias (19) y (20) con autorización. puntuaciones iguales o mayores a 20 requieren claramente una evaluación diagnóstica más amplia de la dependencia de

La encuesta AUDIT fue aplicada por una alumna del último año de la carrera de Ingeniería Estadística de una universidad chilena de viva voz a 419 estudiantes de este centro de estudios, que dieron su consentimiento al responder la encuesta, tras ser informados del objetivo de la investigación. Todos ellos recibieron las instrucciones necesarias para comprender las preguntas. Ninguno estaba intoxicado ni necesitaba tratamiento de urgencia. A todos se les aseguró la confidencialidad de sus respuestas y no existió discriminación de ningún tipo al ser entrevistados. Las condiciones ambientales y de seguridad fueron las adecuadas para realizar la entrevista. Debe subrayarse que la confiabilidad del instrumento no se vio afectada por factores como la homogeneidad del grupo, el tiempo en realizar la encuesta, el tamaño del cuestionario ni la objetividad del proceso de asignación de puntuaciones.

Como los tipos y las cantidades de bebidas alcohólicas varían según la cultura y las costumbres, en Chile, un trago equivale aproximadamente a una lata de cerveza, una copa de vino o un corto de licor (9).

Cabe señalar que se elaboró un informe para el Departamento de Salud de la Institución con el fin de que se tomaran las medidas respectivas y oportunas para tratar a los estudiantes que presentaron síntomas de dependencia o consumo perjudicial de alcohol. se estimaron el alfa de Cronbach y el alfa ordinal considerando como aceptables coeficientes de confiabilidad mayores de alcohol" (19).

Para validar el AUDIT, en este estudio 
0,7 (2), tanto para la encuesta total como para cada uno de sus dominios con el objetivo de evaluar la confiabilidad del instrumento.

Puesto que la finalidad de esta investigación es exhibir las ventajas del alfa ordinal frente al alfa de Cronbach, a continuación se presentan ecuaciones con las cuales se estimaron estos estadísticos.

(a) Mediante la varianza de los ítems (alfa de Cronbach) (2),

$$
\alpha=\frac{n}{n-1}\left(1-\frac{\sum_{i=1}^{n} v_{i}}{v_{t}}\right),
$$

donde $n$ es el número de ítems,

$v_{i}$ es la varianza de cada ítem, $i=1$, $2, \ldots, n$,

$v_{t}$ es la varianza del total.

(b) Mediante la matriz de correlaciones o de correlaciones policóricas (4),

$$
\alpha=\frac{n}{n-1}\left[\frac{n(\bar{\lambda})^{2}-\overline{\lambda^{2}}}{n(\bar{\lambda})^{2}+\overline{u^{2}}}\right],
$$

donde $n$ es el número de ítems, $\bar{\lambda}$, la media aritmética de los pesos factoriales, $\overline{\lambda^{2}}$, la media aritmética de los cuadrados de los pesos factoriales, y $\overline{u^{2}}$, la media aritmética de las unicidades de los pesos factoriales.

Si se utiliza la matriz de correlaciones, se obtiene una estimación del alfa de Cronbach y la versión ordinal del coeficiente alfa se calcularía aplicando esta ecuación sobre la matriz de correlaciones policóricas.

(c) Mediante el mayor valor propio (versión categórica del alfa) (20),

$$
\alpha=\frac{n(\tau-1)}{(n-1) \tau},
$$

donde $n$ es el número de ítems y $\tau$, el mayor valor propio de la matriz de correlación.

Según sus autores (20), esta ecuación maximiza la estimación que se hace del coeficiente alfa de Cronbach, es decir, sobreestima dicho estadístico.

La mayoría de los cálculos se realizaron con el lenguaje de programación $\mathrm{R}$ de uso libre y los restantes, con SPSS o Excel (8). Los códigos fundamentales para la obtención de los resultados en $\mathrm{R}$ son los siguientes (8):

\# Comandos para calcular el alfa Ordinal

library(psych) \# para cargar la

attach(Datos) $\quad$ \# para trabajar solo con los datos

items $<$-data.frame \# para formar la

(P1,P2,P3,P4,P5,P6, matriz con los

$\mathrm{P} 7, \mathrm{P} 8, \mathrm{P9}, \mathrm{P} 10) \quad$ ítems

cor_poly \# calcula la

<-polychoric(items) correlación

policórica

alpha(cor_poly\$rho) \# calcula el alfa Ordinal

\# Comando para calcular el alfa de Cronbach

alpha(items)

\# calcula el alfa de Cronbach

fa(items)

\# calcula las cargas factoriales.

\section{RESULTADOS}

La población del estudio comprendió a 419 estudiantes, distribuidos en dos sedes, con 250 participantes en una sede $(59,7 \%)$ y 169 en la otra $(40,3 \%) ; 259$ fueron hombres $(61,8 \%)$ y 160 , mujeres $(38,2 \%)$. La media de la edad de los encuestados fue de 21,5 años.

En el cuadro 2 se muestran los resultados del análisis descriptivo para cada uno de los ítems (preguntas) de que consta el instrumento AUDIT. Los cálculos se realizaron con Excel.

En el cuadro 3 se presentan las cargas factoriales, valores esenciales con los cuales se puede estimar el coeficiente alfa, en sus dos versiones, utilizando la ecuación dada en (b). En el cuadro 4 aparecen las estimaciones de los coeficientes alfa de Cronbach y alfa ordinal, usando SPSS, R o Excel y utilizando las

\section{CUADRO 2. Medidas de tendencia central y de variación}

\begin{tabular}{lcccc}
\hline Número del ítem & Varianza & Media & Asimetría & Curtosis \\
\hline 1 & 1,11 & 1,59 & 0,09 & $-0,56$ \\
2 & 1,25 & 0,92 & 1,14 & 0,46 \\
3 & 1,05 & 0,97 & 0,77 & $-0,52$ \\
4 & 0,60 & 0,36 & 2,36 & 5,21 \\
5 & 0,34 & 0,22 & 3,31 & 12,90 \\
6 & 0,35 & 0,22 & 3,25 & 11,55 \\
7 & 0,47 & 0,34 & 2,52 & 7,60 \\
8 & 0,41 & 0,35 & 2,18 & 6,02 \\
9 & 1,35 & 0,44 & 2,48 & 4,59 \\
10 & 1,15 & 0,38 & 2,72 & 6,02 \\
Varianzas & Total & Dimensión & Dimensión 2 & Dimensión 3 \\
& 33,52 & 7,70 & 2,33 & 5,73 \\
\hline
\end{tabular}

Fuente: Elaboración propia.

\begin{tabular}{|c|c|c|c|c|c|c|}
\hline \multirow{2}{*}{ Ítem i } & \multicolumn{3}{|c|}{ Matriz de correlación } & \multicolumn{3}{|c|}{ Matriz policórica } \\
\hline & $\lambda_{i}$ & $\lambda_{i}^{2}$ & $u_{i}^{2}$ & $\lambda_{i}$ & $\lambda_{i}^{2}$ & $u_{i}^{2}$ \\
\hline 1 & 0,79 & 0,63 & 0,37 & 0,82 & 0,68 & 0,32 \\
\hline 2 & 0,66 & 0,44 & 0,56 & 0,71 & 0,51 & 0,49 \\
\hline 3 & 0,85 & 0,72 & 0,28 & 0,87 & 0,76 & 0,24 \\
\hline 4 & 0,65 & 0,43 & 0,57 & 0,79 & 0,62 & 0,38 \\
\hline 5 & 0,54 & 0,29 & 0,71 & 0,7 & 0,49 & 0,51 \\
\hline 6 & 0,59 & 0,34 & 0,66 & 0,72 & 0,51 & 0,49 \\
\hline 7 & 0,51 & 0,26 & 0,74 & 0,61 & 0,37 & 0,63 \\
\hline 8 & 0,63 & 0,4 & 0,6 & 0,75 & 0,56 & 0,44 \\
\hline 9 & 0,40 & 0,16 & 0,84 & 0,59 & 0,35 & 0,65 \\
\hline 10 & 0,50 & 0,25 & 0,75 & 0,66 & 0,43 & 0,57 \\
\hline \multirow[t]{2}{*}{ Media } & 0,61 & 0,39 & 0,61 & 0,72 & 0,53 & 0,47 \\
\hline & $\bar{\lambda}$ & $\overline{\lambda^{2}}$ & $\overline{u^{2}}$ & $\bar{\lambda}$ & $\overline{\lambda^{2}}$ & $\overline{u^{2}}$ \\
\hline
\end{tabular}

CUADRO 3. Pesos factoriales y unicidades usando la matriz de correlación y de correlación policórica

Fuente: Elaboración propia. 
CUADRO 4. Coeficientes de confiabilidad para la encuesta AUDIT

\begin{tabular}{|c|c|c|c|c|c|c|c|}
\hline & \multicolumn{5}{|c|}{ Alfa de Cronbach usando } & \multicolumn{2}{|c|}{ Alfa Ordinal usando } \\
\hline & SPSS & $\mathrm{R}$ & (a) & (b) & (c) & (b) & $\mathrm{R}$ \\
\hline Dominio 1 & 0,84 & 0,84 & 0,84 & 0,84 & 0,84 & 0,88 & 0,90 \\
\hline Dominio 2 & 0,67 & 0,67 & 0,67 & 0,68 & 0,67 & 0,81 & 0,81 \\
\hline Dominio 3 & 0,55 & 0,55 & 0,55 & 0,60 & 0,61 & 0,75 & 0,75 \\
\hline Total & 0,84 & 0,84 & 0,84 & 0,85 & 0,86 & 0,91 & 0,92 \\
\hline
\end{tabular}

Fuente: Elaboración propia.

ecuaciones presentadas en los incisos (a), (b) y (c) cuando fue necesario, para mostrar que el alfa ordinal se puede obtener con cálculos relativamente simples (21). Los resultados mostrados bajo las etiquetas (a), (b) y (c) se realizaron con Excel.

\section{DISCUSIÓN}

Los resultados del cuadro 4 son congruentes con lo expresado en la bibliografía y son los esperados en el sentido de que, en el caso del alfa de Cronbach, los valores fueron similares para la encuesta completa y las dimensiones 1 y 2 en todas las estimaciones. Para la dimensión 3, se obtuvieron valores mayores en el caso de las estimaciones usando las ecuaciones presentadas en (b) y (c) que en los resultados obtenidos con $\mathrm{R}$, SPSS y (a), lo que se espera dado que se sabe que la estimación definida en (c) sobreestima el valor de alfa. Al considerar el criterio mínimo de 0,7 , no serían aceptados los dominios 2 y 3 . Respecto al alfa ordinal, los valores obtenidos usando (b) y $\mathrm{R}$ fueron muy similares, aceptando la encuesta total y cada una de las dimensiones.

Los valores del alfa de Cronbach obtenidos en esta investigación (cuadro 4) son muy próximos a los obtenidos en otro estro estudio (17): 0,83 para toda la encuesta y para los dominios 1, 2, y 3, $0,80,0,66$ y 0,62 , respectivamente. En este último estudio citado, la encuesta AUDIT se hizo a una población de estudiantes universitarios al igual que la muestra considerada en este estudio, lo que implica, y se comprueba, que las conclusiones respecto a la fiabilidad en el presente estudio y en el de la referencia 17 deberían ser similares.

Al comparar los resultados de la presente investigación con los obtenidos en otra (22), en la cual hizo un estudio nacional con la población general de Chile, se puede observar que el valor para la encuesta total es muy similar: 0,83 para ese estudio (22) y 0,84 para el presente. Para los dominios 1, 2 y 3 los valores obtenidos en dicho estudio (22) fueron 0,$74 ; 0,81$ y 0,66 respectivamente, con lo cual en él se obtuvieron conclusiones similares a las obtenidas en la presente investigación, excepto en el caso del dominio 2, esto es, en ese estudio se consideraron aceptables los dominios 1 y 2 , y no aceptables, el dominio 3, lo que no coincide con las conclusiones de la presente investigación y del estudio de la referencia 17, en el cual sólo se aceptó el dominio 1 (17).

Los valores mostrados en el cuadro 4 también son congruentes con lo que se esperaba en el sentido de que los valores del alfa de Cronbach subestiman la confiabilidad y de que se encontraron valores menores para el alfa de Cronbach en todos los casos. Destacan sobremanera los dominios 2 y 3 , que presentan valores del alfa de Cronbach menores que el límite aceptable, lo que no sucede al usar el alfa ordinal. Esto implica que, usando el alfa de Cronbach como coeficiente de confiabilidad interna, no hay relación entre las preguntas 4, 5 y 6 ni entre las preguntas 7, 8, 9 y 10. Por el contrario, si se emplea el alfa ordinal, dichas preguntas sí están relacionadas.

Las diferencias entre los valores del alfa de Cronbach y el alfa ordinal pueden deberse a que los valores de asimetría y curtosis para algunas de las preguntas en el cuestionario son altos, y esto influye en el valor de la estimación del alfa de Cronbach $(2,5)$.

En este estudio algunas preguntas de la escala tienen menos de 5 alternativas y se observa que el alfa de Cronbach subestima la confiabilidad del instrumento, lo que es congruente con lo publicado (5). Al trabajar con observaciones cualitativas con pocas alternativas de respuesta, se debe usar el alfa ordinal y no el alfa de Cronbach.
En esta investigación se considera que tanto la encuesta total como los dominios tienen una confiabilidad aceptable, dado que en este caso se prefiere usar el alfa ordinal en detrimento del alfa de Cronbach por las razones mencionadas anteriormente.

Los resultados del cuadro 4 corresponden a una aplicación particular que muestra empíricamente la ventaja de utilizar el alfa ordinal respecto al alfa de Cronbach.

Existen formas alternativas para estimar la consistencia interna de un instrumento que no se han considerado en este estudio, como el método de división por mitades o método de Kuder-Richardson (1). También es importante destacar que FACTOR es otro paquete estadístico de libre disposición $(2,21)$, aunque no se ha utilizado en el presente estudio, con el cual se pueden estimar el alfa de Cronbach y el alfa ordinal de manera relativamente sencilla.

El hecho de considerar un índice que no cumpla los supuestos necesarios para ser utilizado con el fin de tomar decisiones respecto a la confiabilidad de una dimensión puede desembocar en conclusiones erróneas y afectar de manera significativa el desarrollo de una investigación. A este respecto, en este estudio se entregaron los comandos de $\mathrm{R}$ que permiten calcular el alfa ordinal de manera sencilla y se muestran las ventajas de su utilización para evaluar la confiabilidad de encuestas que no cumplen los supuestos necesarios para considerar el alfa de Cronbach.

Agradecimientos. Los autores agradecen a los revisores anónimos y al editor de esta revista su valioso tiempo y sus cuidadosos comentarios y sugerencias con los cuales se ha logrado mejorar la calidad de este artículo.

Conflictos de interés. Los autores declaran no tener conflictos de interés.

Financiación. Este estudio ha recibido financiación del Grupo de Investigación Matemática Aplicada GI 172409/C de la Universidad del Bío-Bío, Concepción, Chile.

Declaración. Los autores son los únicos responsables de las opiniones expresadas en el manuscrito, que no necesariamente reflejan la opinión o política de la RPSP/PAJPH y / o la OPS. 


\section{REFERENCIAS}

1. Aiken L. Tests psicológicos y evaluación. 11ava. ed. México, DF: Pearson Educación; 2003.

2. González J, Pazmino M. Cálculo e interpretación del Alfa de Cronbach para el caso de validación de la consistencia interna de un cuestionario, con dos posibles escalas tipo Likert. Rev Publicando. 2015;2(1):62-77.

3. Trizano-Hermosilla I, Alvarado J. Best Alternatives to Cronbach's Alpha Reliability in Realistic Conditions: Congeneric and Asymmetrical Measurements. Frontiers Psychol. 2016;7(1):769-76.

4. Elosua P, Zumbo B. Coeficientes de fiabilidad para escalas de respuesta categórica ordenada. Psicothema. 2008;20(5):896-901.

5. Gadermann AM, Guhn M, Zumbo BD. Estimating ordinal reliability for Likerttype and ordinal item response data: A conceptual, empirical, and practical guide. Pract Assess Res Eval. 2012;17(4):1-13.

6. Peters GJY. The alpha and the omega of scale reliability and validity: why and how to abandon Cronbach's alpha and the route towards more comprehensive assessment of scale quality. Europ Health Psychol. 2014;16(2):56-69.

7. Zumbo BD, Gadermann AM, Zeisser C. Ordinal versions of coefficients alpha and theta for Likert rating scales. J Modern Appl Stat Meth. 2007;6(1):20-9.

8. $\mathrm{R}$ Core Team. R: A Language and Environment for Statistical Computing. Vienna: R Foundation for Statistical Computing; 2016. Disponible en: http:// www.R-project.org Acceso el 18 de diciembre de 2017.

9. Ministerio de Salud. Consumo perjudicial y Dependencia de Alcohol y otras Drogas en personas menores de 20 años. Santiago de Chile: MINSAL; 2013. Disponible en: http://web.minsal.cl/portal/url/item/ 7222c0667853b8f8e04001011f016146.pdf Acceso el 18 de diciembre de 2017.

10. Adewuya AO. Validation of the alcohol use disorders identification test (audit) as a screening tool for alcohol-related problems among Nigerian university students. Alcohol Alcoholism. 2005;40(7):575-7.

11. Arrieta K. Consumo patológico de alcohol entre los estudiantes de la Universidad de Cartagena. Rev Salud Publica. 2008;11(7):878-86.

12. Díaz A, Díaz L, Hernandez-Ávila C, Narro J, Fernández H, Solís C. Prevalencia del consumo riesgoso y dañino de alcohol y factores de riesgo en estudiantes universitarios de primer ingreso. Salud Mental. 2008;31(5):271-82

13. Guerra A, Vieira P, García L. I Levantamento Nacional Sobre o Uso de Alcool, Tabaco y Otras drogas entre Universitarios de 27 Capitales Brasileiras. Brasilia: SENAD; 2010.

14. Lucas AC, Parente RC, Picanço NS, Conceição DA, Costa KR, Magalhães IR, et al. Use of psychoactive drugs by health sciences undergraduate students at the Federal University in Amazonas. Cad Saude Publica. 2006;22(4):663-71.

15. Matute RC, Pillon SC. Alcohol consumption by nursing students in Honduras. Rev Latinoam Enferm. 2008;16(especial):584-9.

16. Mota N, Álvarez-Gil R, Corral $M$, Rodríguez S, Parada M, Crego A, et al. Risky alcohol use and heavy episodic drinking among Spanish university students: a two-year follow up. Gac Sanit. 2010;24(6):372-7.

17. Seguel F, Santander G, Alexandre O. Validez y confiabilidad del test de identificación de los trastornos debidos al consumo de alcohol (AUDIT) en estudiantes de una universidad chilena. Rev Ciencia y Enfermeria XIX. 2013;19(1):23-35.
18. Álvarez-Hernández G, Delgado-De la Mora J. Diseño de Estudios Epidemiológicos. I. El Estudio Transversal: Tomando una Fotografía de la Salud y la Enfermería. Bol Clin Hosp Infant Edo Son. 2015;32(1):26-34.

19. Babor TF, Higgins-Biddle JC, Saunders JB, Monteiro MG. AUDIT Cuestionario de identificación de los transtornos debidos al consumo de alcohol. Pautas para su utilización en Atención Primaria. Ginebra: Organización Mundial de la Salud; 2001.

20. Meulman J, Van der Kooij A, Heiser W. Principal Components Analysis with Nonlinear Optimal Scaling Transformations for Ordinal and Nominal Data. En: Kaplan D, ed. The Sage Handbook of Quantitative Methodology for the Social Sciences. Thousand Oaks: Sage; 2004: 49-70.

21. Baglin, J. Improving Your Exploratory Factor Analysis for Ordinal Data: A Demonstration Using FACTOR. Pract Assess Res Eval. 2014;19(5):1-15.

22. Donoso MP. Análisis de Resultados del Alcohol Use Disorders Identification Test (AUDIT) Resultados Escala. Bol Observa Chil Drogas. 2015;(3):1-6. Disponible en http:/ / www.senda.gob.cl/media/boletines/Boletin $\% 203 \% 20 \mathrm{An} \% \mathrm{C} 3 \%$ A 1 lisis $\% 20$ de $\% 20$ Resultados $\% 20$ del $\% 20$ Alcohol $\% 20$ Use $\% 20$ Disorders $\%$ 20Identification $\% 20$ Test $\% 20$ (AUDIT) \%20Resultados $\% 20$ Escala.pdf Acceso el 18 de diciembre de 2017.

Manuscrito recibido el 9 de marzo de 2017. Aceptado para publicación, tras revisión, el 16 de julio de 2017.

Objectives. Demonstrate the advantages of using ordinal alpha when the assumptions for Cronbach's alpha are not met; show the usefulness of ordinal alpha with the Chilean version of the WHO Alcohol Use Disorders Identification Test (AUDIT); and provide the commands in $\mathrm{R}$ programming language for performing the respective calculations.

\section{Advantages of ordinal alpha versus Cronbach's alpha, illustrated using the WHO AUDIT test}

Methods. Cronbach's alpha and ordinal alpha were compared in an AUDIT questionnaire administered to 419 students at a Chilean university.

Results. In all domains, the result using ordinal alpha was greater than with Cronbach's alpha. This finding was consistent with the literature on the subject. When ordinal alpha was chosen, the reliability of each domain and the survey as a whole was accepted, which does not always happen with Cronbach's alpha.

Conclusions. In making decisions about the reliability of a dimension, consideration should be given to an index that meets the required assumptions for using it in order to avoid erroneous conclusions that can have an appreciable effect on research. The values of Cronbach's alpha underestimate the reliability of an instrument when working with ordinal scales.

Keywords Alcohol drinking; validation studies; reproducibility of results; Chile. 
RESUMO Objetivos. Apresentar as vantagens do uso do alfa ordinal nos casos em que não são cumpridos os pressupostos do alfa de Cronbach e demonstrar a utilidade do alfa ordinal com a versão chilena do instrumento AUDIT e criar comandos na linguagem de

Vantagens do alfa ordinal em relação ao alfa de Cronbach verificadas na pesquisa AUDIT-OMS

Palavras chave programação R para realizar os respectivos cálculos.

Métodos. Foi realizada a comparação entre o alfa de Cronbach e o alfa ordinal com o uso do instrumento AUDIT aplicado a 419 alunos de uma universidade chilena.

Resultados. Em todos os domínios, o alfa ordinal foi maior que o alfa de Cronbach, o que é condizente com o descrito na literatura. Ao considerar o alfa ordinal, aceita-se a confiabilidade de cada um dos domínios e da pesquisa completa, o que não ocorre completamente quando se usa o alfa de Cronbach.

Conclusões. Para tomar decisões sobre a confiabilidade de uma dimensão, deve-se considerar um índice que cumpra os pressupostos necessários para seu uso, porque do contrário pode-se chegar a conclusões errôneas e afetar consideravelmente o desenvolvimento de uma pesquisa. Os valores do alfa de Cronbach subestimam a confiabilidade do instrumento por se basear em escalas ordinais.

Consumo de bebidas alcoólicas; estudos de validação; reprodutibilidade de resultados; Chile. 